Proyecciones Journal of Mathematics Vol. 31, No 2, pp. 103-123, June 2012. Universidad Católica del Norte Antofagasta - Chile

\title{
Polar topologies on sequence spaces in non-archimedean analysis
}

\author{
R. AMEZIANE HASSANI \\ A. EL AMRANI \\ UNIVERSITÉ SIDI MOHAMED BEN ABDELLAH, MOROCCO \\ and \\ M. BABAHMED UNIVERSITÉ MOULAY ISMAIL, MOROCCO \\ Received : May 2011. Accepted : January 2012
}

\begin{abstract}
The purpose of the present paper is to develop a theory of a duality in sequence spaces over a non-archimedean vector space. We introduce polar topologies in such spaces, and we give basic results characterizing compact, $C$-compact, complete and $A K$-complete subsets related to these topologies.
\end{abstract}

Key words : Locally $K$-convex topologies, non archimedean sequence spaces, Schauder basis, separated duality.

$M S C 2010: 11 F 85-46 A 03-46 A 20-46 A 22-46 A 35-46 A 45-$ $464 A 50$. 


\section{Introduction}

The duality $\left\langle\lambda, \lambda^{\alpha}\right\rangle$, where $\lambda$ is a scalar sequence space, was studied by Köthe and Toeplitz [7] and it has been reformulated by Köthe [6] using the theory of locally convex spaces. After, the duality $\left\langle\lambda, \lambda^{\beta}\right\rangle$ has been studied by Chillingworth [2], Matthews [8], T. Komura and Y. Komura [4]. In this work, we are interested to a duality in non-archimedean sequence spaces. We consider a separated duality $\langle X, Y\rangle$ of vector spaces over a non-archimedean valued field $K(n . a)$; in [1] Ameziane and Babahmed gave a fundamental properties of this duality. Afterwards we take $E(X)$ and $E(Y)$ two vector-valued sequence spaces over $X$ and $Y$ respectively such that $E(Y) \subset E(X)^{\beta}$ that are endwed with the separated duality $\langle E(X), E(Y)\rangle$ by the canonic bilinear form (p.108). We introduce the notion of polar topoogies over $E(X)$; and by the linear maps $\pi_{j}^{X}$ and $\delta_{j}^{X}$ which we define in this paper; we study the polar topologies compatible with the duality $\langle E(X), E(Y)\rangle$ using the basic duality $\langle X, Y\rangle$. Finally we characterize $C$ - compact, $A K$-complete and complete subsets of $E(X)$ relatively at these topologies. This study was useful in the study that we made in [3].

Throughout this paper, $K$ is a non-archimedean $(n . a)$ non trivially valued complete field with valuation |.|, $X$ and $Y$ are two $n . a$ topological vector spaces over $K$ (or $K$ vector spaces) that are in separated duality $\langle X, Y\rangle$. The duality theory for locally $K$-convex spaces can be found more extensively in [1], [9] , [11] and [12].

\section{Preliminary}

A nonempty subset $A$ of a $K$-vector space $X$ is called $K$-convex if $\lambda x+$ $\mu y+\gamma z \in A$ whenever $x, y, z \in A, \lambda, \mu, \gamma \in K,|\lambda| \leq 1,|\mu| \leq 1,|\gamma| \leq 1$ and $\lambda+\mu+\gamma=1$. $A$ is said to be absolutely $K$-convex if $\lambda x+\mu y \in A$ whenever $x, y \in A, \lambda, \mu \in K,|\lambda| \leq 1,|\mu| \leq 1$. For a nonempty set $A \subset X$ its $K$-convex hull $c(A)$ and absolutely $K$-convex hull $c_{0}(A)$ are respectively the smallest $K$-convex and absolutely $K$-convex set that contains $A$. If $A$ is a finite set $\left\{x_{1}, \ldots, x_{n}\right\}$ we sometimes write $c_{0}\left(x_{1}, \ldots, x_{n}\right)$ instead of $c_{0}(A)$.

An absolutely $K$-convex subset of a locally $K$-convex space $X$ is called $K$ - closed if for every $x \in X$ the set $\{|\lambda|: \lambda \in K, \lambda x \in A\}$ is closed in $|K|$. If the valuation on $K$ is discrete every absolutely $K$-convex set $A$ is $K$-closed. If $K$ has a dense valuation an absolutely $K$ convex set $A$ is 
$K$-closed if and only if from $x \in E, \lambda x \in A$ for all $\lambda \in K,|\lambda| \prec 1$ it follows that $x \in A$. Intersections of $K$-closed sets are $K$-closed. For an absolutely $K$-convex set $A$ the $K$-closed hull of $A$ is the smallest subset of $X$ that is $K$-closed and contains $A$, it is denoted by $K_{c}(A)$. If $K$ is discrete we have $K_{c}(A)=A$ and if $K$ is dense, $K_{c}(A)=\cap\{\lambda A: \lambda \in K$ and $|\lambda| \succ 1\}$ ([1] p. 220).

A topological vector space $X$ over $K$ is called locally $K$-convex space if $X$ has a base of zero consisting of locally $K$-convex sets.

Let $(X, \tau)$ a locally $K$-convex space, $\tau$ is define by a family of n.a. semi-norms $\tau$ - continuous over $X$, and if $K$ is discrete, we can suppose that $N_{p}=\{p(x) / x \in X\} \subset|K|$ for every $p \in \mathcal{P}([9])$; where $(\mathcal{P})$ is a family of $n . a$ semi-norms which define the topology $\tau$.

If $p$ is a $(n . a)$ semi-norm over $X, B_{p}(0,1)$ is the set $\{x \in X: p(x) \leq 1\}$.

A sequence $\left(e_{i}\right)_{i}$ is a Schauder basis for $X$ if every $x \in X$ can be written uniquely as $x=\sum_{i=1}^{\infty} \lambda_{i} x_{i}$ where the coefficient functionals $f_{j}: x \longmapsto \lambda_{j}$ are continuous.

Let $X$ a $K$-vector space and $M$ a subset of $X$, a $K$-convex filter over $M$, is a filter $\mathcal{F}$ over $M$ having a basis $\mathcal{B}$ consisting of $K$-convex subsets of $M$; this basis is called $K$-convex basis of $K$-convex filter $\mathcal{F}$.

The order of all filters on $M$ induces an order on all $K$-convex filters on $M$. A maximal element of the ordered set of $K$-convex filter on $M$ is called maximal $K$-convex filter of $M$.

Let $\left(x_{i}\right)_{i \in I}$ a net on $M$; for all $i \in I$, put $F_{i}=\left\{x_{j} / j \geq i\right\} .\left(F_{i}\right)_{i \in I}$ is a filter over $M$ called filter associated to a net $\left(x_{i}\right)_{i \in I}$. Conversely, if $\mathcal{F}=\left(F_{i}\right)_{i \in I}$ is a filter over $M$, for all $i \in I$ let $x_{i} \in F_{i}$; over $I$ we define the following order: $i \leq j \Leftrightarrow F_{j} \subset F_{i} .\left(x_{i}\right)_{i \in I}$ is a net in $M$ called a net associated to a filter $\mathcal{F}$.

Proposition 1. Let $X$ a locally $K$-convex space, $M$ a subset of $X$ and $\mathcal{F}=\left(F_{i}\right)_{i \in I}$ a maximal $K$-convex filter over $M$.

1. $\mathcal{F}$ converges or not having any clusterpoint .

2. Let $\left(x_{i}\right)_{i \in I}$ a net associated to a $\mathcal{F}$; if $\left(x_{i}\right)_{i \in I}$ converges to $x_{0}, \mathcal{F}$ converges to $x_{0}$.

Proof. 1. Let $x_{0}$ a cluster point of $\mathcal{F}$ and $\left(U_{j}\right)_{j \in J}$ a $K$-convex neighbourhood base of $x_{0}, \mathcal{F}^{\prime}=\left\{F_{i} \cap U_{j} / i \in I\right.$ and $\left.j \in J\right\}$ is a $K$-convex filter which converges to $x_{0}$ and it is coarsest than $\mathcal{F}$, then $\mathcal{F}=\mathcal{F}^{\prime}$.

2. $x_{0}$ is a clusterpoint of $\left(x_{i}\right)_{i \in I}$, then it is a clusterpoint of $\mathcal{F}$, and so $\mathcal{F}$ converges to $x_{0}$. 
Proposition 2. Let $X, Y$ two $K$-vector spaces, $f: X \longrightarrow Y$ a linear map and $\mathcal{F}=\left(F_{i}\right)_{i \in I}$ a maximal $K$-convex filter over $X$ that having $\mathcal{B}$ us a $K$-convex basis; $f(\mathcal{B})$ is a $K$-convex basis of a maximal $K$-convex filter over $Y$.

A subset $A$ of a locally $K$-convex space $X$ is compactoid if for each neighbourhood $U$ of zero there exist $x_{1}, \ldots, x_{n} \in X$ such that $A \subset U+$ $c_{0}\left(x_{1}, \ldots, x_{n}\right)$. An absolutely $K$-convex subset $A$ of $X$ is said to be $C$-compact if every convex filter on $A$ has a clusterpoint on $A$.

$K$ is $C$-compact if and only if $K$ is spherically complete.

Proposition 3. Let $M$ be a subset of $X$. The following are equivalent:

(i). $M$ is $C$-compact;

(ii). Every maximal $K$-convex filter over $M$ converges;

(iii). Any family of closed and $K$-convex subsets of $M$ whose intersection is empty contains a finite subfamily whose intersection is empty.

Let $\mathcal{B}$ a basis of a filter $\mathcal{F}$ on a subset $M$ of $X$; the smallest $K$-convex filter containing $\mathcal{B}$, is called $K$-convex filter generated by $\mathcal{B}$ and is denoted by $\mathcal{F}_{c}(\mathcal{B})$. We show that $\mathcal{F}_{c}(\mathcal{B})=\{F \subset M /$ there exists $B \in \mathcal{B}: c(B) \subset F\}$, and $c(\mathcal{B})$ is $K$-convex basis of $\mathcal{F}_{c}(\mathcal{B})$, that is to say $\mathcal{F}_{c}(\mathcal{B})=\mathcal{F}(c(\mathcal{B}))$.

If $\left(x_{i}\right)_{i \in I}$ is a net in $X ;\left(x_{i}\right)_{i \in I}$ converges to $x_{0}$ if and only if the filter $K$-convex associated with $\left(x_{i}\right)_{i \in I}$ converges to $x_{0}$.

Proposition 4. Let $X, Y$ two $K$-vector spaces, $f: X \longrightarrow Y$ a linear map, $M$ a subset of $X$ and $\mathcal{B}$ a base of filter on $M$. Then $f(\mathcal{B})$ is a base of filter on $f(M)$, and we have $\mathcal{F}_{c}(f(\mathcal{B}))=f\left(\mathcal{F}_{c}(\mathcal{B})\right)$.

$\left(\omega(X), \tau_{\omega}(X)\right)=$ the linear space of all sequences in $X$ endowed with the product topology $\tau_{\omega}(X)$ which is generated by the family of n.a seminorms $\left(p_{n}\right)_{n \in \mathbb{N}, p \in(\mathcal{P})}, p_{n}(\bar{x})=p\left(x_{n}\right)$ for all $\bar{x}=\left(x_{n}\right)_{n} \in \omega(X)$ and all $p \in(\mathcal{P})$, if $X$ is a locally $K$-convex space and $(\mathcal{P})$ is a family of n.a semi-norms which define his topology; this space is noted $\omega(K)$ (or $\omega$, for short) in case when $X=K$. A sequence space over $X$ is a subspace of $\omega(X)$.

We define the following sequence spaces over $X$

$$
\begin{aligned}
& c_{0}(X)=\left\{\left(x_{k}\right)_{k} \in \omega(X):\left(x_{k}\right)_{k} \text { converges to zero }\right\} \\
& c(X)=\left\{\left(x_{k}\right)_{k} \in \omega(X):\left(x_{k}\right)_{k} \text { converges in } X\right\} \\
& \varphi(X)=\left\{\left(x_{k}\right)_{k} \in \omega(X): \text { there exists } k_{0} \in \mathbb{I}: x_{k}=0 \text { for all } k \geq k_{0}\right\}, \\
& m(X)=\left\{\left(x_{k}\right)_{k} \in \omega(X):\left(x_{k}\right)_{k} \text { is bounded in } X\right\} .
\end{aligned}
$$


Over $m(X)$ we define the sequence of n.a semi-norms $(\bar{p})_{p \in(\mathcal{P})}$ by:

$\bar{p}(\bar{x})=\sup _{k} p\left(x_{k}\right)$ for all $\bar{x}=\left(x_{k}\right)_{k} \in m(X)$.

Let $\tau_{\infty}(X)$ be the topology on $m(X)$ defined with the sequence of n.a semi-norms $(\bar{p})_{p \in(\mathcal{P})}$.

\section{Polar topologies}

Let $X$ and $Y$ two $K$-vector spaces placed in separating duality $\langle X, Y\rangle$. If $A$ is a subset of $X$, we denote by $A^{\circ}=\{y \in Y /|\langle x, y\rangle| \leq 1 \quad$ for all $x \in A\}$ the polar of $A$ and $A^{\circ \circ}=\left\{x \in X /|\langle x, y\rangle| \leq 1\right.$ for all $\left.y \in A^{\circ}\right\}$ the bipolar of $A$.

$A^{\circ}$ is absolutely $K$-convex and $\sigma(Y, X)$-bounded.

For each absolutely $K$-convex subset $A$ of $Y, K_{c}\left(\bar{A}^{\sigma(Y, X)}\right)=A^{\circ \circ}([1]$, corollary 4.3, p. 233). A subset $A$ of $Y$ is said to be $X$-closed if for every $y \in Y \backslash A$, there exits $x \in X$ such that $|\langle x, y\rangle| \succ 1$ and $|\langle x, A\rangle| \leq 1$. Intersections of $X$-closed sets are $X$-closed. For a subset $A$ of $Y$ the $X$-closed hull $X_{c}(A)$ of $A$ is the smallest $X$-closed subset of $Y$ that contains $A$. For each subset $A$ of $Y, X_{c}(A)=A^{\circ \circ}([1]$, proposition 2.5 , p. $224)$. Using these two results and by [1], theorem 4.2 , p. 233 we have: for all absolutely $K$-convex subset $A$ of $Y, A$ is $X$-closed, if and only if, $A$ is $K$-closed and $\sigma(Y, X)$-closed.

Let $\mathcal{A}$ be a family of $\sigma(Y, X)$-bounded subsets of $Y$ such that

(a) $\mathcal{A}$ is directed by inclusion,

(b) $Y=\bigcup_{A \in \mathcal{A}} A$

(c) there exists $\lambda_{0} \in K,\left|\lambda_{0}\right|>1$ such that $\lambda_{0} A \in \mathcal{A}$, for all $A \in \mathcal{A}$.

A topology $\tau$ on $X$ is called polar topology of $\mathcal{A}$-convergence, if $\tau$ has a fundamental system of zero-neighbourhood (F.S.N) consisting of $\left\{A^{\circ} / A \in \mathcal{A}\right\}$.

A vector topology $\tau$ on $X$ is called polar topology if there exists a family $\mathcal{A}$ of $\sigma(Y, X)$-bounded subsets of $Y$ which has the properties $(a),(b)$ and (c), such that $\tau$ is a polar topology of $\mathcal{A}$-convergence. it is defined by the family of n.a. semi-norms $\left(P_{A}\right)_{A \in \mathcal{A}}$, where $P_{A}(x)=\sup \{|\langle x, y\rangle| / y \in A\}$.

If $\mathcal{A}$ is the family of all subsets of $Y$ that are:

1. Absolutely $K$-convex, weakly bounded and weakly $C$-compacts, we have the $C$-compact topology $\tau_{c}(X, Y)=\tau_{c}$,

2. Absolutely convex and $\sigma(Y, X)$-compact, we have the Mackey topology $\tau_{m}(X, Y)=\tau_{m}$, 
3. $\sigma(Y, X)$-bounded and $X$-closed, we have the $X$-closed topology $\tau_{e}(X, Y)=\tau_{e}$.

4. $\sigma(Y, X)$-bounded, we have the strong topology $\tau_{b}(X, Y)$.

A locally $K$-convex topology $\tau$ on $X$ is called compatible with the duality $\langle X, Y\rangle$ or $(X, Y)$-compatible if $Y$ is isomorphic to the topological dual of $X$ provided with the topology $\tau$. The weak topology $\sigma(X, Y)$ is the coarsest topology among all topologies $(X, Y)$-compatible, and the upper bound topology of all topologies $(X, Y)$-compatible topology is the finest among all the topologies $(X, Y)$-compatible.

We say that $X$ is semi-reflexive if $X$ is isomorphic to the strong topological dual of $Y$ and if $\tau$ is a locally $K$-convex topology on $X$ we say that $X$ is $\tau$-reflexive if $X$ is semi-reflexive and $\tau=\tau_{b}\left(X, X^{\prime}\right)$.

For further information about polar topology of $\mathcal{A}$-convergence and general properties of locally $K$-convex spaces we refer to [1], [11] and [12].

If $A \subset \omega(X)$, the $\beta$-dual of $A$ is the subspace of $\omega(Y)$ which is define by $A^{\beta}=\left\{\left(y_{n}\right)_{n} \in \omega(Y): \lim _{n}\left\langle x_{n}, y_{n}\right\rangle=0\right.$ for all $\left.\left(x_{n}\right)_{n} \in A\right\} . A$ is called perfect if $A^{\beta \beta}=A$. If $A$ is perfect then $\varphi(X) \subset A$. For all $A \subset \omega(X), A^{\beta}$ is perfect. We define $B^{\beta}$ if $B \subset \omega(Y)$ on the same way.

A subset $D$ of $\omega(X)$ is said to be solid if for every $\bar{x}=\left(x_{k}\right)_{k} \in D$ and $\alpha=\left(\alpha_{k}\right)_{k} \in \omega$ such that $\left|\alpha_{k}\right| \leq 1$ for all $k$, we have $\alpha \bar{x}=\left(\alpha_{k} x_{k}\right)_{k} \in D$. The solid hull $S(D)$ of $D$ is the smallest solid set of sequence containing $D$.

A topology on $E(X)$, with respect the duality $\left\langle E(X), E(X)^{\beta}\right\rangle$, will be called solid if the elements of the determining family of weakly bounded subsets of $E(X)^{\beta}$ are solids sets.

Let $E(X)$ and $E(Y)$ be two sequence spaces on $X$ and $Y$ respectively such that $E(Y) \subset E(X)^{\beta}$, we define on the pair $(E(X), E(Y))$ the following duality $\left\langle\left(x_{n}\right)_{n},\left(y_{n}\right)_{n}\right\rangle=\sum_{n=1}^{\infty}\left\langle x_{n}, y_{n}\right\rangle$ for all $\left(x_{n}\right)_{n} \in E(X)$ and all $\left(y_{n}\right)_{n} \in E(Y)$.

If $\varphi(X) \subset E(X)$ and $\varphi(Y) \subset E(Y)$, the duality $\langle E(X), E(Y)\rangle$ is separate.

In the sequel $\langle E(X), E(Y)\rangle$ denotes a duality of this type.

$S(E(Y)) \subset[S(E(X))]^{\beta}$ and $\langle S(E(X)), S(F(Y))\rangle$ is a separating duality extending the separating duality $\langle E(X), F(Y)\rangle$, therefore, we can assume that $E(X)$ and $F(Y)$ are solid.

For all $j \geq 1$, we consider the following linear mappings: 


$$
\begin{aligned}
& \pi_{j}^{X}: E(X) \longrightarrow X \quad \delta_{j}^{X}: X \longrightarrow E(X) \\
& \left(x_{n}\right) \longrightarrow x_{j} \quad a \longrightarrow \delta_{j}(a)
\end{aligned}
$$

where $\delta_{j}(a)$ is the sequence with $a$ in the j-th place and 0's elsewhere.

We define also $\pi_{j}^{Y}$ and $\delta_{j}^{Y}$.

Let $x=\left(x_{k}\right) \in \omega(X)$, for all $n \geq 1 \quad x^{[n]}=\sum_{j=1}^{n} \delta_{j}\left(x_{j}\right)$ is called the $n^{i t h}$ section of $x$.

We have: $\pi_{j}^{X} o \delta_{j}^{X}=i d_{X}, \pi_{j}^{Y} o \delta_{j}^{Y}=i d_{Y},\left(\pi_{j}^{X}\right)^{*} / Y=\delta_{j}^{Y}$ and $\left(\delta_{j}^{X}\right)^{*} / F(Y)=$ $\pi_{j}^{Y}$ where $u^{*}$ is the algebraic adjoint of the linear map $u$.

Proposition 5. Let $A$ be a subset of $E(X)$ if $A$ is solid, $A^{\circ}$ is solid and we have: $A^{\circ}=[A \cap \varphi(X)]^{\circ}$.

Definition 1. Let $A$ a subset of $\omega(X)$.

a. Is said that $A$ is $\delta_{j}^{X}$-saturated if for all $\left(x_{n}\right) \in A, \delta_{j}^{X}\left(x_{j}\right) \in A$.

$b$. It is said that $A$ is $\delta^{X}$-saturated if $A$ is $\delta_{j}^{X}$-saturated for all $j \geq 1$.

c. It is said that $A$ is $\pi^{X}$-saturated if: $x_{j} \in \pi_{j}^{X}(A)$ for all $j \geq 1 \Rightarrow$ $\left(x_{n}\right) \in A$.

If $A$ is solid, $A$ is $\delta^{X}$-saturated.

$\varphi(X)$ is $\delta^{X}$-saturated and not $\pi^{X}$-saturated.

If $p$ is a n.a. semi-norm on $X,\left\{\left(x_{n}\right) \in \omega(X) / \sup _{n} p\left(x_{n}\right) \leq 1\right\}$ is $\pi^{X}$-saturated.

The following results are demonstrated in a direct:

Proposition 6. Let $A$ be a subset of $E(X)$.

1. If $A$ is $\pi^{X}$-saturated, $S(A)$ is $\pi^{X}$-saturated.

2. If $A$ is $\delta^{X}$-saturated, $S(A)$ and $c_{0}(A)$ are $\delta^{X}$-saturated, and $A^{\circ}$ is $\delta^{Y}-$ saturated and $\pi^{Y}$-saturated.

3. $\left[\pi_{j}^{X}(A)\right]^{\circ} \subset \pi_{j}^{Y}\left(A^{\circ}\right)$ for all $j \geq 1$.

4. If $A$ is $\delta_{j}^{X}$-saturated, $\left[\pi_{j}^{X}(A)\right]^{\circ}=\pi_{j}^{Y}\left(A^{\circ}\right)$.

5. If $A$ is $\delta^{X}$-saturated,

$A^{\circ}=\pi_{j}^{X}\left[\pi_{j}^{Y}\left(A^{\circ}\right)\right]=\left\{\left(y_{k}\right) \in F(Y) / \sup _{k}\left|\left\langle x_{k}, y_{k}\right\rangle\right| \leq 1 \quad\right.$ for all $\left.\left(x_{k}\right) \in A\right\}$.

6. $S(A)^{\circ} \subset S\left(A^{\circ}\right)$; and if $A$ is $\delta^{X}$-saturated, $A^{\circ}=S(A)^{\circ}=S\left(A^{\circ}\right)$. 
7. If $A$ is $\delta^{X}$-saturated and $F(Y)$-closed, $\pi_{j}^{X}(A)$ is $Y$-closed for all $j \geq 1$.

8. If $A$ is $\pi^{X}$-saturated and $\pi_{j}^{X}(A)$ is $Y$-closed for all $j \geq 1$, $A$ is $F(Y)-$ closed.

Corollary 1. Let $A$ be a subset of $E(X) \delta^{X}$-saturated and $\pi^{X}$-saturated.

For $A$ is $F(Y)$-closed, it is necessary and enough that $\pi_{j}^{X}(A)$ be $Y$-closed for all $j \geq 1$.

Proposition 7. Let $A$ be an absolutely $K$-convex subset of $E(X)$.

1. If $A$ is $K$-closed and $\delta_{j}^{X}$-saturated, $\pi_{j}^{X}(A)$ is $K$-closed.

2. If $A$ is $\pi^{X}$-saturated and $\pi_{j}^{X}(A)$ is $K$-closed for all $j \geq 1$, $A$ is $K$-closed.

Proposition 8. Let $\tau$ be a topology on $E(X)$ and $\tau_{j}$ the topology image reciprocal of $\tau$ by the linear map $\delta_{j}^{X}$ on $X$. If $\tau$ admits as S.F.N of $0\left\{A^{\circ} / A \in \mathcal{A}\right\}$, then $\left\{\left[\pi_{j}^{Y}(A)\right]^{\circ} / A \in \mathcal{A}\right\}$ is a F.S.N. of 0 for $\tau_{j}$.

Proof. ([1], proposition 2.9).

Proposition 9. For all $j \geq 1, \pi_{j}^{X}$ is $(\sigma(E(X), F(Y)), \sigma(X, Y))$-continuous and $\delta_{j}^{X}$ is $(\sigma(X, Y), \sigma(E(X), F(Y)))$-continuous.

Proof. $\quad\left(\pi_{j}^{X}\right)^{*}(Y) \subset F(Y)$ and $\left(\delta_{j}^{X}\right)^{*}(F(Y)) \subset Y$, and the result follows from $([9], p .128)$.

Proposition 10. 1. $\left[\pi_{j}^{X}(A)\right]^{\circ}=\left(\delta_{j}^{Y}\right)^{-1}\left(A^{\circ}\right)$ for all $A \subset E(X)$.

2. $\left[\delta_{j}^{X}(B)\right]^{\circ}=\left(\pi_{j}^{Y}\right)^{-1}\left(B^{\circ}\right)$ for all $B \subset X$.

3. $\pi_{j}^{X}(A) \subset B \Rightarrow \delta_{j}^{Y}\left(B^{\circ}\right) \subset A^{\circ}$ for all $A \subset E(X)$ and for all $B \subset X$.

4. $\delta_{j}^{X}(B) \subset A \Rightarrow \pi_{j}^{Y}\left(A^{\circ}\right) \subset B^{\circ}$ for all $A \subset E(X)$ and for all $B \subset X$.

5. $\left(\pi_{j}^{X}\right)^{-1}\left(D^{\circ}\right)=\left[\delta_{j}^{Y}(D)\right]^{\circ}$ for all $D \subset Y$.

6. $\left(\delta_{j}^{X}\right)^{-1}\left(C^{\circ}\right)=\left[\pi_{j}^{Y}(C)\right]^{\circ}$ for all $C \subset F(Y)$.

7. $\left(\pi_{j}^{X}\right)^{*}(D) \subset C \Rightarrow \pi_{j}^{X}\left(C^{\circ}\right) \subset D^{\circ}$ for all $D \subset Y$ and for all $C \subset E(Y)$.

8. $\left(\delta_{j}^{X}\right)^{*}(C) \subset D \Rightarrow \delta_{j}^{X}\left(D^{\circ}\right) \subset C^{\circ}$ for all $D \subset Y$ and for all $C \subset E(Y)$.

Proof. ([1], proposition 2.8). 
A polar topology of $\mathcal{A}$-convergence on $E(X)$ is said solid, if all $A \in \mathcal{A}$ is solid. Thus, any polar, solid topology admits a F.S.N from 0 consisting of solid subsets .

If $\tau$ is the polar topology of $\mathcal{A}$-convergence on $E(X)$ such that $A$ is $\delta^{Y}$-saturated for all $A \in \mathcal{A}, \tau$ coincides with the polar topology of $S(\mathcal{A})$-convergence (proposition 6), and then $\tau$ is a polar and solid topology

Proposition 11. Let $\tau$ be a polar topology of $\mathcal{A}$-convergence over $E(X)$ and $\tau_{j}$ the topology image reciprocal of $\tau$ by the linear map $\delta_{j}^{X}$ on $X$.

1. $\tau_{j}$ is the polar topology of $\pi_{j}^{Y}(\mathcal{A})$-convergence.

2. $\pi_{j}^{X}$ is $\left(\tau, \tau_{j}\right)$-continuous if and only if $\delta_{j}^{Y}$ o $\pi_{j}^{Y}(A) \in \mathcal{A}$ for all $A \in \mathcal{A}$.

Proof. ([1], proposition 3.8$)$.

Proposition 12. If $\tau$ is the weak topology (resp. Mackey, resp. C-compact, resp.

$E(X)$-closed; resp. strong) of $E(X)$ for all $j \geq 1, \tau_{j}$ is the weak topology (resp. Mackey, resp. $C$-compact, resp. $X$-closed; resp. strong) on $X$

Proof. ([1], proposition 3.9$)$.

Proposition 13. Let $\tau$ a polar topology of $\mathcal{A}$-convergence on $E(X)$, for all $j \geq 1$, we have:

1. $\delta_{j}^{X}$ is $\left(\tau_{j}, \tau\right)$-continuous;

2. If $\tau$ is solid, $\pi_{j}^{X}$ is $\left(\tau, \tau_{j}\right)$-continuous;

3. If $\pi_{j}^{X}$ is $\left(\tau, \tau_{j}\right)$-continuous, $\delta_{j}^{X}$ is $\left(\tau_{j}, \tau\right)$-closed.

Proof. 1. $\tau_{j}$ is a polar topology of $\pi_{j}^{Y}(\mathcal{A})$-convergence, and we have:

$\delta_{j}^{X}\left(\left[\pi_{j}^{Y}(A)\right]^{\circ}\right) \subset A^{\circ}$ for all $A \in \mathcal{A}$.

2. If $\tau$ is solid, we have : $\pi_{j}^{X}\left(A^{\circ}\right) \subset\left[\pi_{j}^{Y}(A)\right]^{\circ}$ for all $A \in \mathcal{A}$.

3. Let $M$ a closed in $\left(X, \tau_{j}\right)$, there exists $A \in \mathcal{A}$ such that $\left[\pi_{j}^{Y}(A)\right]^{\circ} \subset$ $M^{\circ}$, therefore $A^{\circ} \subset \delta_{j}^{X}\left(M^{\circ}\right)=\left[\delta_{j}^{X}(M)\right]^{\circ}$.

Let $\tau$ be a locally $K$-convex topology on $E(X)$ such that $E(X)$ be $\tau$-polar; if $\tau$ is $(E(X) F(Y))$-compatible, $\tau$ is a polar topology of $\mathcal{A}$-convergence, where $\mathcal{A}$ is constituted of $\sigma(F(Y), E(X))$-bounded and 
$E(X)$-closed subsets of $F(Y)$, ([1 ], theorem 4.3). For all $j \geq 1, \tau_{j}$ is the polar topology of $\pi_{j}^{Y}(\mathcal{A})$-convergence on $X$ and $X$ is $\tau_{j}$-polar if all $A \in \mathcal{A}$ is $\delta^{Y}$-saturated, $\pi_{j}^{X}(A)$ is $\sigma(Y, X)$-bounded and $X$-closed (Proposition $6)$, and then $\tau_{j}$ is $(X, Y)$-compatible.

If $K$ is spherically complete, we have the following theorem:

Theorem 1. Suppose that $K$ be spherically complete, and let $\tau$ a locally $K$-convex topology on $E(X)$; if $\tau$ is $(E(X), F(Y))$-compatible, $\tau_{j}$ is $(X, Y)$-compatible, for all $j \geq 1$.

Proof. $\quad \tau$ is a polar topology of $\mathcal{A}$ convergence, where $\mathcal{A}$ consists of absolutely $K$ convex, $\sigma(E(Y), E(X))$-bounded and $\sigma(E(Y), E(X))$ $C$-compact subsets of $F(Y)$ ([1], theorem 4.4). For all $j \geq 1, \pi_{j}^{Y}$ is $(\sigma(F(Y), E(X)), \sigma(Y, X))$-continuous, then $\pi_{j}^{Y}(A)$ is absolutely $K$-convex, $\sigma(Y, X)$ - bounded and $\sigma(Y, X)-C$-compact for all $A \in \mathcal{A}$ and then $\tau_{j}$ is $(X, Y)$-compatible.

Theorem 2. Let $\tau$ a solid and polar topology on $E(X)$; if $E(X)$ is $\tau$-barreled, $X$ is $\tau_{j}$-barreled for all $j \geq 1$.

Proof. Let $B$ a $\tau_{j}$-barrel in $X ; \delta_{j}^{X}$ is $\left(\tau_{j}, \tau\right)$-closed, then $\delta_{j}^{X}(B)$ is a $\tau$ - barrel into $E(X)$ and then $\left(\delta_{j}^{X}\right)^{-1}\left(\delta_{j}^{X}(B)\right)$ is a neighborhood of 0 in $\left(X, \tau_{j}\right)$ then $B$ is a neighborhood of 0 for $\tau_{j}$.

Remark 1. Instead of assuming that $\tau$ is solid, we can assume only that $\pi_{j}^{X}$ be $\left(\tau, \tau_{j}\right)$-continuous for all $j \geq 1$.

A subset $A$ of $E(X)$ said to be $\delta^{X}$-stable if for all $x=\left(x_{k}\right) \in E(X)$ such that there exists $j \geq 1$ satisfying $\delta_{j}^{X}\left(x_{j}\right) \in A$, then $x \in A$. stable.

Let $A \subset E(X)$ such that $A \cap\left\{\delta_{j}^{X}(a) / a \in X\right.$ and $\left.j \geq 1\right\}=\phi, A$ is $\delta^{X}$

Definition 2. Let $\tau$ a vector topology on $E(X)$; we say that $E(X)$ is $\delta^{X} \tau$-barreled if every $\tau$-barrel $\delta^{X}$-stable, is a neighborhood of 0 .

If $E(X)$ is $\tau$-barreled, it is $\delta^{X} \tau$-barreled.

Theorem 3. Let $\tau$ a polar and solid topology on $E(X)$; if there exists $j \geq$ 1 such that $X$ is $\tau_{j}$-barreled, $E(X)$ is $\delta^{X} \tau$-barreled 
Proof. Let $B$ a $\tau$-barrel $\delta^{X}$-stable in $E(X) ; \delta_{j}^{X}$ is $\left(\tau_{j}, \tau\right)$-continuous, so $\left(\delta_{j}^{X}\right)^{-1}(B)$ is a $\tau_{j}$-barrel, and then $\left(\delta_{j}^{X}\right)^{-1}(B)$ is a neighborhood of 0 in $\left(X, \tau_{j}\right)$ and hence $\left(\pi_{j}^{X}\right)^{-1}\left[\left(\delta_{j}^{X}\right)^{-1}(B)\right]$ is a neighborhood of 0 in $(E(X), \tau) . B$ is $\delta^{X}$ - stable, then $\left(\pi_{j}^{X}\right)^{-1}\left[\left(\delta_{j}^{X}\right)^{-1}(B)\right] \subset B$ and then $B$ is a neighborhood of 0 in $(E(X), \tau)$.

Theorem 4. Suppose that $X$ and $Y$ are semi-reflexive, and let $\tau$ a topology on $E(X)$ which is $(E(X), F(Y))$-compatible. If $E(X)$ is $\tau$-reflexive, $X$ is $\tau_{j}$-reflexive for every $j \geq 1$.

Proof. $\quad \tau=\tau_{b}\left(E(X), E(X)^{\prime}\right)=\tau_{b}(E(X), F(Y))$; so for all $j \geq 1 \tau_{j}=$ $\tau_{b}(X, Y)$ (Proposition 12). $Y$ is semi-reflexive, then $\tau_{j}$ is $(X, Y)$-compatible ([1], proposition 5.9) and then $\tau_{j}=\tau_{b}\left(X,\left(X, \tau_{j}\right)^{\prime}\right)$.

Corollary 2. If $K$ is spherically complete and $\tau$ is a topology on $E(X)$ which is $(E(X), F(Y))$-compatible and solid such that $E(X)$ is $\tau$-barreled, then $X$ is $\tau_{j}$ reflexive for any $j \geq 1$.

Proof. For all $j \geq 1, \tau_{j}$ is $(X, Y)$-compatible (theorem 1 ) and $X$ is $\tau_{j}$-barreled for all $j \geq 1$, then $X$ is $\tau_{j}$-reflexive ([1], theorem 5.2).

\section{Compactness and $C$-compactness}

Let $\tau$ a polar topology on $E(X)$ such that $\pi_{j}^{X}$ be $\left(\tau, \tau_{j}\right)$-continuous for all $j \geq 1$. If $M$ is a compact subset of $(E(X), \tau) ; \pi_{j}^{X}(M)$ is a compact subset of $\left(X, \tau_{j}\right)$ for all $j \geq 1$.

In order to study the converse, we introduce the notion of $T K$-convergent net.

Definition 3. A net $\left(x^{i}\right)_{i \in I}$ in $E(X)$ is called $T K$-convergent if for all $j \geq 1,\left(x_{j}^{i}\right)_{i \in I}$ is convergent in $\left(X, \tau_{j}\right)$.

Theorem 5. Let $M$ a subset of $E(X) ; M$ is relatively compact in $(E(X), \tau)$ if and only if:

(i.) $\pi_{j}^{X}(M)$ is relatively compact in $\left(X, \tau_{j}\right)$ for all $j \geq 1$;

(ii.) All $T K$-convergent net in $M$, converges in $(E(X), \tau)$. 
Proof. N.C.] $\pi_{j}^{X}$ is $\left(\tau, \tau_{j}\right)$-continuous for all $j \geq 1$, then $\pi_{j}^{X}(M)$ is relatively compact in $\left(X, \tau_{j}\right)$. Let $\left(x^{i}\right)_{i \in I}$ a $T K$-convergent net in $M$. For all $j \geq 1$ let $x_{j} \in X$ such that $\left(x_{j}^{i}\right)_{i \in I}$ converges to $x_{j}$ in $\left(X, \tau_{j}\right) .\left(x^{i}\right)_{i \in I}$ has a cluster point $z=\left(z_{n}\right)$ in $(E(X), \tau)$. For all $j \geq 1, z_{j}$ is a cluster point of $\left(x_{j}^{i}\right)_{i \in I}$ in $\left(X, \tau_{j}\right)$; then $z_{j}=x_{j} .\left(x_{n}\right)$ is the unique cluster point of $\left(x^{i}\right)_{i \in I}$, therefore $\left(x^{i}\right)_{i \in I}$ converges to $\left(x_{n}\right)$ in $(E(X), \tau)$.

S.C.] Let $\left(x^{i}\right)_{i \in I}$ a net in $M$, and let $\mathcal{A}$ the family of $\sigma(F(Y), E(X))$-bounded subset of $F(Y)$ which defines the topology $\tau$. For any $j \geq 1, \tau_{j}$ is the polar topology of $\pi_{j}^{Y}(\mathcal{A})$-convergence on $X$.

Let $x_{1}$ a cluster point of $\left(x_{1}^{i}\right)_{i \in I}$ in $\left(X, \tau_{1}\right)$. For all $A \in \mathcal{A}$ and for all $i \in I$, there exists $i_{A}>i$ such that $x_{1}^{i_{A}} \in\left[\pi_{1}^{Y}(A)\right]^{\circ}$. Consider the sub family $\left(i_{A}\right)_{A \in \mathcal{A}}$ of $I$, it is ordered by: $i_{A} \leq i_{B} \Leftrightarrow A \subset B$ for all $A, B \in \mathcal{A}$. $\left(i_{A}\right)_{A \in \mathcal{A}}$ is a filter on the right family. Let $A_{0} \in \mathcal{A} ; i_{A} \geq i_{A_{0}} \Rightarrow A_{0} \subset$ $A \Rightarrow\left[\pi_{1}^{Y}(A)\right]^{\circ} \subset\left[\pi_{1}^{Y}\left(A_{0}\right)\right]^{\circ} \Rightarrow x_{1}^{i_{A}}-x_{1} \in\left[\pi_{1}^{Y}\left(A_{0}\right)\right]^{\circ}$. Therefore $\left(x_{1}^{i_{A}}\right)_{A \in \mathcal{A}}$ converges to $x_{1}$ in $\left(X, \tau_{1}\right)$.

Let $x_{2}$ a cluster point of $\left(x_{2}^{i_{A}}\right)_{A \in \mathcal{A}}$ in $\left(X, \tau_{2}\right)$. for all $A \in \mathcal{A}$, there exists $l_{1}\left(i_{A}\right)>i_{A}$ such that $x_{2}^{l_{1}\left(i_{A}\right)}-x_{2} \in\left[\pi_{2}^{Y}(A)\right]^{\circ}$.

Let $A_{0} \in \mathcal{A} ; i_{A} \geq i_{A_{0}} \Rightarrow A \supset A_{0} \Rightarrow\left[\pi_{2}^{Y}(A)\right]^{\circ} \subset\left[\pi_{2}^{Y}\left(A_{0}\right)\right]^{\circ} \Rightarrow x_{2}^{l_{1}\left(i_{A}\right)}-$ $x_{2} \in\left[\pi_{2}^{Y}\left(A_{0}\right)\right]^{\circ}$. Therefore $\left(x_{2}^{l_{1}\left(i_{A}\right)}\right){ }_{A \in \mathcal{A}}$ converges to $x_{2}$ in $\left(X, \tau_{2}\right)$. Let $x_{3}$ a cluster point of $\left(x_{3}^{l_{1}\left(i_{A}\right)}\right)_{A \in \mathcal{A}}$ in $\left(X, \tau_{3}\right)$. For all $A \in \mathcal{A}$, there exists $l_{2}\left(l_{1}\left(i_{A}\right)\right)>l_{1}\left(i_{A}\right)$ such that $x_{3}^{l_{2} o l_{1}\left(i_{A}\right)}-x_{3} \in\left[\pi_{3}^{Y}(A)\right]^{\circ} \cdot\left(x_{3}^{l_{2} o l_{1}\left(i_{A}\right)}\right)_{A \in \mathcal{A}}$ converges to $x_{3}$ in $\left(X, \tau_{3}\right)$.

Inductively, for all $j \geq 3$ and for all $A \in \mathcal{A}$, there exists $l_{j} o l_{j-1} o \ldots . . l_{1}\left(i_{A}\right)>$ $l_{j-1} o \ldots . . o l_{1}\left(i_{A}\right)$ such that $\left(x_{j+1}^{l_{j} o \ldots o l_{1}\left(i_{A}\right)}\right)_{A \in \mathcal{A}}$ converges to $x_{j+1}$ in $\left(X, \tau_{j+1}\right)$.

Put $y=\left(x^{i_{A}}, x^{l_{1}\left(i_{A}\right)}, x^{l_{2} o l_{1}\left(i_{A}\right)}, \ldots ., x^{l_{k} o \ldots o l_{1}\left(i_{A}\right)}, \ldots .\right)_{A \in \mathcal{A}}$.

For all $j \geq 1,\left(x_{j}^{i_{A}}, x_{j}^{l_{1}\left(i_{A}\right)}, x_{j}^{l_{2} o l_{1}\left(i_{A}\right)}, \ldots ., x_{j}^{l_{k} o \ldots o l_{1}\left(i_{A}\right)}, \ldots\right)_{A \in \mathcal{A}}$ converges to $x_{j}$ in $\left(X, \tau_{j}\right)$; therefore $y$ is $T K$-convergent, and hence it converges to $x$ in $(E(X), \tau)$. Hence $x$ is a cluster point of $\left(x^{i}\right)_{i \in I}$, and then $M$ is relatively compact.

Corollary 3. Let $M$ a subset of $E(X), M$ is compact in $(E(X), \tau)$ if and only if:

(i.) $\pi_{j}^{X}(M)$ is compact in $\left(X, \tau_{j}\right)$ for all $j \geq 1$,

(ii.) Any $T K$-convergent net in $M$ converges to an element of $M$ in $(E(X), \tau)$.

To give version of theorem 5 using the filters, we need introduce the 
following definition:

Definition 4. Let $M$ a subset of $E(X)$ and $\mathcal{F}$ a filter on $M$; we say that $\mathcal{F}$ is $T K$ - convergent if for all $j \geq 1$ the filter generated by $\pi_{j}^{X}(\mathcal{F})$ converges in $\left(X, \tau_{j}\right)$.

Every convergent filter is $T K$-convergent, and if $\mathcal{F}$ is a $T K$-convergent filter and $\mathcal{F}^{\prime}$ is a filter finer than $\mathcal{F}, \mathcal{F}^{\prime}$ is $T K$-convergent.

Proposition 14. Let $M$ a subset of $E(X)$.

1. If $\mathcal{F}=\left(F_{i}\right)_{i \in I}$ is a $T K$-convergent filter on $M$, any net associated to $\mathcal{F}$ is $T K$-convergent.

2. If $\left(x^{i}\right)_{i \in I}$ is a TK-convergent net, the $K$-convex filter associated to $\left(x^{i}\right)_{i \in I}$ is $T K$-convergent.

Theorem 6. Let $M$ a subset of $E(X) ; M$ is compact in $(E(X), \tau)$ if and only if:

(i.) $\pi_{j}^{X}(M)$ is compact in $\left(X, \tau_{j}\right)$ for all $j \geq 1$;

(ii.) Any $T K$-convergent filter on $M$ converges to an element of $M$.

Proof. N.C.] Let $\mathcal{F}$ a $T K$-convergent filter on $M$. For any $j \geq 1$ let $x_{j} \in X$ such that $\pi_{j}^{X}(\mathcal{F})$ converges to $x_{j}$ in $\left(X, \tau_{j}\right) . \mathcal{F}$ has at least one cluster point $z=\left(z_{n}\right)$ in $M$. For all $j \geq 1, z_{j}$ is a cluster point of $\pi_{j}^{X}(\mathcal{F})$, therefore $z_{j}=x_{j}$; then $\left(x_{n}\right)$ is the unique cluster point of $\mathcal{F}$ in $M$, so $\mathcal{F}$ converges to $\left(x_{n}\right)$ in $(M, \tau)$.

S.C.] Let $\mathcal{F}$ a maximal filter on $M$; for all $j \geq 1 \pi_{j}^{X}(\mathcal{F})$ is a maximal filter on $\pi_{j}^{X}(M)$, therefore it converges to $x_{j}$ in $\left(X, \tau_{j}\right)$, and then $\mathcal{F}$ is $T K-$ convergent, therefore it converges to an element of $M$.

Definition 5. Let $M$ a subset of $E(X)$, we say that $M$ is an $A K$-complete subset of $(E(X), \tau)$ if every $x=\left(x_{n}\right)$ element of $E(X)$ such that $\left(x^{[n]}\right)$ is a Cauchy sequence in $(M, \tau) ; x \in M$ and $\left(x^{[n]}\right)$ converges to $x$ in $(E(X), \tau)$.

We say that $M$ is relatively $A K$-complete if its closure $\bar{M}$ in $(E(X), \tau)$ is $A K$ - complete.

If $M$ is complete, it is $A K$-complete.

Any closed subset of a set $A K$-complete is $A K$-complete.

In the following result, we characterize the subsets solid and relatively compact of $(E(X), \tau)$. 
Theorem 7. Let $M$ a solid subset of $E(X), M$ is relatively compact in $(E(X), \tau)$ if and only if:

(i.) $\pi_{j}^{X}(M)$ is relatively compact in $\left(X, \tau_{j}\right)$ for all $j \geq 1$,

(ii.) $x \stackrel{[i]}{\stackrel{i \rightarrow \infty}{\longrightarrow} x}$ uniformly on $M$ in $(E(X), \tau)$,

(iii.) $M$ is relatively $A K$-complete in $(E(X), \tau)$.

Proof. N.C.] If $M$ is relatively compact, $M$ is relatively complete, and then it is relatively $A K$-complete.

Suppose we $\operatorname{did} \operatorname{not}(i i$.$) there exists A \in \mathcal{A}$ a sequence $\left({ }^{i} x\right)_{i}$ in $M$ and a strictly increasing sequence of integers $\left(j_{i}\right)_{i}$ such that ${ }^{i} x^{\left[j_{i}\right]}-{ }^{i} x \notin A^{\circ}$ for all $i \geq 1$. The sequence $\left({ }^{i} x^{\left[j_{i}\right]}-{ }^{i} x\right)_{i}$ is $T K$-convergent to 0 , so it converges to 0 in $(E(X), \tau)$ which is absurd.

S.C.] Let $\left({ }^{\alpha} x\right)_{\alpha \in D}$ a net in $M$ such that for all $j \geq 1\left({ }^{\alpha} x_{j}\right)_{\alpha \in D}$ converges to $x_{j}$ in $\left(X, \tau_{j}\right)$. Let $A \in \mathcal{A}$ for all $i \geq 1{ }^{\alpha} x^{[i]}-x^{[i]}=\sum_{n=1}^{i} \delta_{n}^{X}\left({ }^{\alpha} x_{n}-x_{n}\right) \in A^{\circ}$ for $\alpha$ sufficiently large. So for all $i \geq 1{ }^{\alpha} x^{[i]} \stackrel{\alpha}{\longrightarrow} x^{[i]}$ in $(E(X), \tau)$ in particular $x^{[i]} \in \bar{M}$ for all $i \geq 1$. Using this convergence and (ii), we can choose $\alpha$ as $x^{[i]}-x^{[j]}=\left(x^{[i]}-{ }^{\alpha} x^{[i]}\right)+\left({ }^{\alpha} x^{[i]}-{ }^{\alpha} x\right)+\left({ }^{\alpha} x-{ }^{\alpha} x x^{[j]}\right)+\left({ }^{\alpha} x^{[j]}-x^{[j]}\right) \in$ $A^{\circ}$ for $i, j$ sufficiently great. Therefore $\left(x^{[i]}\right)$ is a Cauchy net in $\bar{M}$ and then $x \stackrel{[i]}{\stackrel{i \rightarrow+\infty}{\longrightarrow}} x$ in $(E(X), \tau)$. From this convergence and (ii), we can choose $i$ such that ${ }^{\alpha} x-x=\left({ }^{\alpha} x-{ }^{\alpha} x^{[i]}\right)+\left({ }^{\alpha} x^{[i]}-x^{[i]}\right)+\left(x^{[i]}-x\right) \in A^{\circ}$ for $\alpha$ Large enough, so $\left({ }^{\alpha} x\right)_{\alpha \in D}$ converges to $x$ in $(E(X), \tau)$ and hence $M$ is relatively compact (theorem 5).

Corollary 4. Let $M$ a solid subset of $E(X) ; M$ is compact in $(E(X), \tau)$ if and only if:

(i.) $\pi_{j}^{X}(M)$ is compact in $\left(X, \tau_{j}\right)$ for all $j \geq 1$,

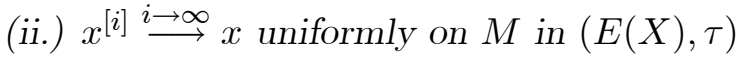

(iii.) $M$ is $A K$-complete in $(E(X), \tau)$.

Corollary 5. The envelope solid of a relatively compact subset of $(E(X), \tau)$ is not necessarily relatively compact.

Proof. Let $x=\left(x_{n}\right) \in E(X)$ such that $\left(x^{[i]}\right)_{i}$ does not converge to $x$ in $(E(X), \tau)$ so $\left(z^{[i]}\right)_{i}$ does not converge to $z$ uniformly on $S(x)$ and then $S(x)$ is not relatively compact.

Proposition 15. 1. Let $\left(x^{i}\right)_{i \in I}$ a net in $E(X)$; if $\mathcal{F}$ is a $K$-convex filter associated with $\left(x^{i}\right)_{i \in I}, \pi_{j}^{X}(\mathcal{F})$ is a $K$-convex filter associated with a net $\left(x_{j}^{i}\right)_{i \in I}$ for all $j \geq 1$. 
2. Let $\mathcal{F}$ a $K$-convex filter on $E(X)$; if $\left(x^{i}\right)_{i \in I}$ is a net associated to $\mathcal{F},\left(x_{j}^{i}\right)_{i \in I}$ is a net associated to $\pi_{j}^{X}(\mathcal{F})$ for all $j \geq 1$.

Theorem 8. Let $M$ a $K$-convex subset of $E(X) ; M$ is $C$-compact in $(E(X), \tau)$ if and only if:

(i.) $\pi_{j}^{X}(M)$ is $C$-compact in $\left(X, \tau_{j}\right)$ for all $j \geq 1$,

(ii.) Any $K$-convex and $T K$-convergent filter on $M$ admits a cluster point in $M$.

Proof. N.C.] Obvious.

S.C.] Let $\mathcal{F}$ a maximum $K$-convex filter of $M$. For any $j \geq 1, \pi_{j}^{X}(\mathcal{F})$ is a maximum $K$-convex filter of $\pi_{j}^{X}(M)$ (proposition 2), so $\pi_{j}^{X}(\mathcal{F})$ converges to $x_{j}$ in $\left(X, \tau_{j}\right) . \mathcal{F}$ is then $T K$-convergent, so it admits a cluster point in $M$, and hence $\mathcal{F}$ converges in $(E(X), \tau)$ (Proposition 1).

Proposition 16. Let $M$ a $K$-convex subset of $E(X)$; if $M$ is $C$-compact, any $K$-convex and $T K$-convergent filter on $M$ has a unique cluster point in $M$.

Proof. Let $\mathcal{F}$ a $K$-convex and $T K$-convergent filter on $M$. For all $j \geq 1$ let $x_{j} \in X$ such that $\pi_{j}^{X}(\mathcal{F})$ converges to $x_{j}$ in $\left(X, \tau_{j}\right) . \mathcal{F}$ admits at least one cluster point $\left(z_{n}\right)$ in $M$. For all $j \geq 1, z_{j}$ is a cluster point of $\pi_{j}^{X}(\mathcal{F})$ in $\left(X, \tau_{j}\right)$, and then $x_{j}=z_{j}$. So $\left(x_{j}\right)$ is the only cluster point of $\mathcal{F}$ in $M$.

5. $A K$-completion and completion

Let $M$ a subset of $E(X)$ and $\tau$ a topology on $E(X)$, we put:

$$
S_{M}=\left\{x \in M / x^{[n]} \stackrel{n \rightarrow \infty}{\longrightarrow} x \text { in }(E(X), \tau)\right\} .
$$

If $M$ is a subspace of $E(X)$, we say that $M$ is an $A K$-space if $S_{M}=M$.

Proposition 17. Let $\tau$ a polar topology of $\mathcal{A}$ convergence on $E(X) ;(E(X), \tau)$ is $A K$-complete.

Proof. Let $x=\left(x_{n}\right) \in E(X)$ such that $\left(x^{[n]}\right)$ is a Cauchy sequence in $(E(X), \tau)$. For all $A \in \mathcal{A}$ there exists $n_{0} \geq 1$ such that $x^{[n]}-x^{[m]} \in A^{\circ}$ for all $n \geq m \geq n_{0}$, and then $x^{[n]}-x \in A^{\circ}$ for all $n \geq n_{0}$, then $x^{[n]} \stackrel{n \rightarrow \infty}{\longrightarrow} x$ in $(E(X), \tau)$. 
Corollary 6. Let $M$ a subset of $E(X) . M$ is $A K-$ complete if and only if $M$ contains every element $x$ of $E(X)$ such that $\left(x^{[n]}\right)$ is the Cauchy sequence in $M$.

Corollary 7. Let $\tau^{\prime}$ a locally $K$-convex topology on $E(X)$ coarser than $\tau$; any $A K$-complete subset of $\left(E(X), \tau^{\prime}\right)$ is complete in $(E(X), \tau)$.

Proof. Let $M$ an $A K$-complete subset of $\left(E(X), \tau^{\prime}\right)$, and either $x \in E(X)$ such that $\left(x^{[n]}\right)$ is a Cauchy sequence in $(M, \tau),\left(x^{[n]}\right)$ is a Cauchy sequence in $\left(M, \tau^{\prime}\right)$, so $x \in M$ and hence $M$ is $A K$-complete in $(E(X), \tau)$, (Corollary 6).

For all $x=\left(x_{n}\right) \in E(X)$, we put $\begin{aligned} \psi_{x}: & E(Y) \longrightarrow c_{0}(K) \\ & \left(y_{n}\right) \longrightarrow\left(\left\langle x_{n}, y_{n}\right\rangle\right)_{n}\end{aligned}$ $\psi_{x}$ is a linear map.

Lemma 1. For any $x \in E(X), \psi_{x}$ is $\left(\sigma(E(Y), E(X)), \sigma\left(c_{0}(K), m(K)\right)\right)-$ continuous.

Proof. $\quad c_{0}(K)^{\beta}=m(K)$ and $\left\langle c_{0}(K), m(K)\right\rangle$ is a separating duality. Let $\left(\alpha_{n}\right) \in m(K) ; E(X)$ is solid, then $\left(\alpha_{n} x_{n}\right) \in E(X)$, and we have $\psi_{x}\left(\left\{\left(\alpha_{n} x_{n}\right)\right\}^{\circ}\right) \subset\left\{\left(\alpha_{n}\right)\right\}^{\circ}$.

Proposition 18. $(E(X), \sigma(E(X), E(Y)))$ is an $A K$-space.

Proof. Let $x=\left(x_{n}\right) \in E(X)$. For all $y=\left(y_{n}\right) \in E(Y),\left(\left\langle x_{n}, y_{n}\right\rangle\right) \in$ $c_{0}(K)$; there exists $i_{0} \geq 1$ such that $\sup _{n \geq i_{0}}\left|\left\langle x_{n}, y_{n}\right\rangle\right| \leq 1$, then $x^{[i]}-x \in\{y\}^{\circ}$ for all $i \geq i_{0}$, and then $x \stackrel{[i]}{\stackrel{i \rightarrow \infty}{\longrightarrow}} x$ in $(E(X), \sigma(E(X), E(Y)))$.

Proposition 19. Suppose that $K$ be local, and let $\tau$ a $(E(X), F(Y))-$ compatible topology on $E(X)$; if $\tau$ is solid, $(E(X), \tau)$ is an $A K-$ space.

Proof. Let $\mathcal{A}$ a family of $\sigma(F(Y), E(X))$-compacts and absolutely $K$-convex subsets of $F(Y)$ such that $\tau$ be a polar topology of $\mathcal{A}$-convergence ([1] , theorem 4.5.) Let $x=\left(x_{n}\right) \in E(X)$; for all $A \in \mathcal{A}, \psi_{x}(A)$ is solid and $\sigma\left(c_{0}(K), m(K)\right)$-compact in $c_{0}(K)$. Then $z^{[i]} \stackrel{i \rightarrow \infty}{\longrightarrow} z$ uniformly on $z \in \psi_{x}(A)$ in $\left(c_{0}(K), \sigma\left(c_{0}(K), m(K)\right)\right.$ ) (theorem 7 ); there exists $i_{0} \geq 1$ such that $\left|\left\langle z^{[i]}-z, e\right\rangle\right| \leq 1$ for all $i \geq i_{0}$ and for all $z \in \psi_{x}(A)$, then

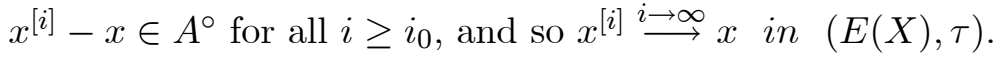

We have the following result which is a kind of reciprocal of theorem 1: 
Theorem 9. Suppose that $K$ be local, and let $\tau$ a polar and solid topology on $E(X)$ for separating duality $\left\langle E(X), E(X)^{\beta}\right\rangle$. If $\tau_{j}$ is $(X, Y)$-compatible for all $j \geq 1, \tau$ is $\left(E(X), E(X)^{\beta}\right)$-compatible.

Proof. $\quad E(X)^{\beta}=\left(E(X), \sigma\left(E(X), E(X)^{\beta}\right)\right)^{\prime} \subset(E(X), \tau)^{\prime}$. Let $f \in$ $(E(X), \tau)^{\prime}$ and $x=\left(x_{n}\right) \in E(X) .(E(X), \tau)$ is an $A K$-space (proposition 19), therefore $x^{[i]} \stackrel{i \rightarrow \infty}{\longrightarrow} x$ in $(E(X), \tau)$, and then $f(x)=\lim _{i} f\left(x^{[i]}\right)=$ $\sum_{j} f o \delta_{j}^{X}\left(x_{j}\right)$. For all $j \geq 1, f o \delta_{j}^{X} \in\left(X, \tau_{j}\right)^{\prime}=Y$; therefore $f(x)=$ $\sum_{j}\left\langle x_{j}, y_{j}\right\rangle$, with $y_{j}=f o \delta_{j}^{X}$ for all $j \geq 1$. Hence $\left(y_{j}\right) \in E(X)^{\beta}$, and so $(E(X), \tau)^{\prime} \subset E(X)^{\beta}$.

Let $\mathcal{C}$ a family of subsets of $F(Y)$ such that:

1. $\mathcal{C}$ is the right filtering for inclusion;

2. There exist $\lambda_{0} \in K,\left|\lambda_{0}\right|>1$ such that $\lambda_{0} A \in \mathcal{C}$ for all $A \in \mathcal{C}$;

3. $\pi_{j}^{Y}(A)$ is $\sigma(Y, X)$-bounded for all $j \geq 1$ and for all $A \in \mathcal{C}$

4. The subspace of $E(Y)$ generated by $\cup\{A / A \in \mathcal{C}\}$ contains $\varphi(Y)$.

We put: $\left\{\begin{array}{l}\mathcal{C}(X)=\left\{\left(x_{n}\right) \in \omega(X) / \sup _{\left(y_{n}\right) \in A}\left|\sum_{n}\left\langle x_{n}, y_{n}\right\rangle\right|<\infty \text { for all } A \in \mathcal{C}\right\} \\ \mathcal{C}(Y)=\text { subspace generated by } \cup\{A / A \in \mathcal{C}\} .\end{array}\right.$

If $\mathcal{C}$ is the family of all finite subsets of $F(Y), \mathcal{C}(X)=F(Y)^{\beta}$.

$\varphi(X) \subset \mathcal{C}(X)$ and $\langle\mathcal{C}(X), \mathcal{C}(Y)\rangle$ is a separating duality defined by the bilinear form:

$\left\langle\left(x_{n}\right),\left(y_{n}\right)\right\rangle=\sum_{n}\left\langle x_{n}, y_{n}\right\rangle$ for all $\left(x_{n}\right) \in \mathcal{C}(X)$ and for all $\left(y_{n}\right) \in \mathcal{C}(Y)$.

If $\tau$ is the polar topology of $\mathcal{A}$-convergence of $E(X),\left(\mathcal{A}(X), \tau_{\mathcal{A}}\right)$ is defined, where $\tau_{\mathcal{A}}$ is the polar topology defined on $\mathcal{A}(X)$ by the family $\mathcal{A}$, and we have:

1. $E(X) \subset \mathcal{A}(X) \subset F(Y)^{\beta}$

2. $\tau_{\mathcal{A} / E(X)}=\tau$.

Proposition 20. Let $\tau$ a polar topology of $\mathcal{A}$-convergence on $E(X)$.

1. $S_{\left(\mathcal{A}(X), \tau_{\mathcal{A}}\right)} \subset E(X)$,

2. $\left(\mathcal{A}(X), \tau_{\mathcal{A}}\right)$ is AK-complete.

Proof. 1. Let $x=\left(x_{n}\right) \in S_{\left(\mathcal{A}(X), \tau_{\mathcal{A}}\right)} ; x^{[i]} \stackrel{i \rightarrow \infty}{\longrightarrow} x\left(\tau_{\mathcal{A}}\right)$, therefore $\left(x^{[i]}\right)$ is Cauchy sequence in $(E(X), \tau)\left(\tau=\tau_{\mathcal{A} / E(X)}\right)$, and then $x \in E(X)$ (proposition $17)$. 
2. Let $\left(x^{[i]}\right)$ a Cauchy sequence in $\left(\mathcal{A}(X), \tau_{\mathcal{A}}\right)$; for all $A \in \mathcal{A}$, there exists $i_{0} \geq 1$ such that for all $i, j \geq i_{0} \sup \left\{\left|\sum_{n=i+1}^{j}\left\langle x_{n}, y_{n}\right\rangle\right| /\left(y_{n}\right) \in A\right\} \leq 1$. We have on the one hand, $\sup \left\{\left|\sum_{n>i_{0}}\left\langle x_{n}, y_{n}\right\rangle\right| /\left(y_{n}\right) \in A\right\} \leq 1$, therefore $\sup \left\{\left|\sum_{n}\left\langle x_{n}, y_{n}\right\rangle\right| /\left(y_{n}\right) \in A\right\}<\infty(\varphi(X) \subset \mathcal{A}(X))$, and then $x \in \mathcal{A}$ $(X)$; on the other hand, for all $i \geq i_{0} \sup \left\{\left|\sum_{n=i+1}^{\infty}\left\langle x_{n}, y_{n}\right\rangle\right| /\left(y_{n}\right) \in A\right\} \leq 1$, therefore $\sup \left\{\left|\left\langle x^{[i]}-x,\left(y_{n}\right)\right\rangle\right| /\left(y_{n}\right) \in A\right\} \leq 1$, and then $x^{[i]} \stackrel{i \rightarrow \infty}{\longrightarrow} x\left(\tau_{\mathcal{A}}\right)$.

Theorem 10. Let $\tau$ a solid and polar topology of $\mathcal{A}$-convergence on $E(X)$. For $E(X)$ is a closed subspace of $\left(\mathcal{A}(X), \tau_{\mathcal{A}}\right)$ it is necessary and sufficient that any Cauchy net $T K$-convergent of $E(X)$ converges in $(E(X), \tau)$.

Proof. N.C.] $A$ is solid for all $A \in \mathcal{A}$, therefore $A^{\circ}=[A \cap \varphi(X)]^{\circ}$.

Let $\left(x^{i}\right)_{i \in I}$ a Cauchy and $T K$-convergent net in $(E(X), \tau)$. For all $j \geq 1$, let $x_{j} \in X$ such that $\left(x_{j}^{i}\right)_{i \in I}$ converges in $\left(X, \tau_{j}\right)$ to $x_{j} . \tau_{j}$ is the polar topology of $\pi_{j}^{Y}(\mathcal{A})$-convergence on $X$. Let $A \in \mathcal{A}$, there exists $k_{0} \in I$ such that for all $r, s \geq k_{0}\left|\sum_{j=1}^{N}\left\langle x_{j}^{r}-x_{j}^{s}, y_{j}\right\rangle\right| \leq 1$ for all $N \geq 1$ and for all $y \in A$. There exists $k_{j} \in I$ such that for all $r \geq k_{j},\left|\left\langle x_{j}^{r}-x_{j}, y_{j}\right\rangle\right| \leq 1$ for all $\left(y_{n}\right) \in A$. Let $r_{0}=\max \left\{k_{0}, k_{1}, \ldots, k_{N}\right\}$ for all $r \geq r_{0}$ we have: $\left|\sum_{j=1}^{N}\left\langle x_{j}^{r}-x_{j}, y_{j}\right\rangle\right| \leq \max _{1 \leq j \leq N}\left|\left\langle x_{j}^{r}-x_{j}, y_{j}\right\rangle\right| \leq 1$ for all $\left(y_{n}\right) \in A$.

$\left|\sum_{j=1}^{N}\left\langle x_{j}^{s}-x_{j}, y_{j}\right\rangle\right| \leq 1$ for all $\left(y_{n}\right) \in A$ and for all $s \geq r_{0}$; therefore $x^{s}-x \in[A \cap \varphi(X)]^{\circ}$ for all $s \geq r_{0}$. Furthermore, $x=x^{s}-\left(x^{s}-x\right) \in \mathcal{A}(X)$. Therefore $\left(x^{i}\right)_{i \in I}$ converges to $x$ in $\left(\mathcal{A}(X), \tau_{\mathcal{A}}\right)$, and then $x \in E(X)$ and $\left(x^{i}\right)_{i \in I}$ converges to $x$ in $(E(X), \tau)$.

S.C.] Let $\left(x^{i}\right)_{i \in I}$ a net in $E(X)$ which converges to $x$ in $\left(\mathcal{A}(X), \tau_{\mathcal{A}}\right) .\left(x^{i}\right)_{i \in I}$ is a Cauchy and $T K$-convergent net in $(E(X), \tau)\left(\tau=\tau_{\mathcal{A} / E(X)}\right)$, therefore $\left(x^{i}\right)_{i \in I}$ converges to $x$ in $(E(X), \tau)$. 
Lemma 2. Let $L$ and $M$ two $K$ - vector spaces, $\tau$ a topology on $L, L \stackrel{\pi}{\longrightarrow}$ $M \stackrel{\delta}{\longrightarrow} L$ two linear maps such as $\pi o \delta=i d_{M}$, and $\tau_{\delta}$ the inverse image topology of $\tau$ by $\delta$ on $M$.

The application $\psi:\left(M, \tau_{\delta}\right) \longrightarrow(\delta(M), \tau), x \longrightarrow \delta(x)$, is an homeomorphism.

Proof. If $\mathcal{U}$ is a F.S.N of 0 for $\tau$; a F.S.N of 0 for $\tau_{\delta}$ is $\delta^{-1}(\mathcal{U})=$ $\left\{\delta^{-1}(U) / U \in \mathcal{U}\right\}$, and we have: $\psi^{-1}(U \cap \delta(M))=\delta^{-1}(U)$ for all $U \in \mathcal{U}$.

Theorem 11. Let $\tau$ a polar and solid topology of $\mathcal{A}$-convergence on $E(X) ;(E(X), \tau)$ is complete if and only if:

(i.) $\left(X, \tau_{j}\right)$ is complete for all $j \geq 1$;

(ii.) $E(X)$ is a closed subspace of $\left(\mathcal{A}(X), \tau_{\mathcal{A}}\right)$.

Proof. N.C.] $\delta_{j}^{X}$ is $\left(\tau, \tau_{j}\right)$-closed for all $j \geq 1$ (proposition 13), therefore $\delta_{j}^{X}(X)$ is a closed subspace of $(E(X), \tau)$, hence $\left(\delta_{j}^{X}(X), \tau\right)$ is complete. Now $\left(\delta_{j}^{X}(X), \tau\right) \simeq\left(X, \tau_{j}\right)$ (lemma 2$)$, therefore $\left(X, \tau_{j}\right)$ is complete. Furthermore $E(X)$ is a closed subspace of $\left(\mathcal{A}(X), \tau_{\mathcal{A}}\right)$ (theorem 10).

S.C.] Let $\left(x^{i}\right)_{i \in I}$ a Cauchy net in $(E(X), \tau)$. For $j \geq 1,\left(x_{j}^{i}\right)_{i \in I}$ is Cauchy in $\left(X, \tau_{j}\right)$ so it converges, and then $\left(x^{i}\right)_{i \in I}$ is $T K$-convergent in $(E(X), \tau)$ so it converges in $(E(X), \tau)$, (theorem 10).

Remark 2. We can replace (ii) of theorem 11 by:

(ii) Any Cauchy $T K$-convergent net in $(E(X), \tau)$ converges in $(E(X), \tau)$.

Corollary 8. Let $\tau$ a polar and solid topology of $\mathcal{A}$-convergence on $E(X)$. If $E(X)$ is a closed subspace of $\left(\mathcal{A}(X), \tau_{\mathcal{A}}\right) ;(E(X), \tau)$ is sequentially complete if and only if $\left(X, \tau_{j}\right)$ is sequentially complete for all $j \geq 1$.

Lemma 3. Let $\tau$ a vector topology on $E(X)$; if $\tau$ is solid, $S_{E(X)}$ is the closure of $\varphi(X)$ in $(E(X), \tau)$.

Proof. $\quad S_{E(X)} \subset \overline{\varphi(X)}$. Let $x=\left(x_{n}\right) \in \overline{\varphi(X)}$ and $U$ a solid neighborhood of 0 , it is $z=\left(z_{n}\right) \in \varphi(X)$ as $x-z \in U$. Since $U$ is solid $x^{[i]}-x \in U$ for $i$ large enough, then $x \stackrel{[i]}{\stackrel{i \rightarrow \infty}{\longrightarrow}} x$ in $(E(X), \tau)$ and hence $x \in S_{E(X)}$.

Proposition 21. Let $\tau$ a solid and polar topology of $\mathcal{A}$-convergence on $E(X)$; if $\left(X, \tau_{j}\right)$ is complete for all $j \geq 1,\left(S_{E(X)}, \tau\right)$ is complete. 
Proof. $\quad S_{E(X)}=\overline{\varphi(X)}$ (lemma 3), therefore $\left(S_{E(X)}, \tau\right)$ is a closed subspace of $\left(\mathcal{A}(X), \tau_{\mathcal{A}}\right)$, and then $\left(S_{E(X)}, \tau\right)$ is complete.

Application: Let $(X,\|\|$.$) a n . a$ Banach space, we consider $m(X)$ endowed with the n.a. norm $\|\cdot\|_{\infty}$. We have $c_{0}(X)=S_{m(X)}$, and $\|\cdot\|_{\infty}$ defines a polar and solid topology on $m(X)$, therefore $\left(c_{0}(X),\|\cdot\|_{\infty}\right)$ is complete.

Theorem 12. Let $\tau$ a solid and polar topology of $\mathcal{A}$-convergence on $E(X)$; if $E(X)$ is an $A K$-space, $(E(X), \tau)$ is complete if and only if $\left(X, \tau_{j}\right)$ is complete for all $j \geq 1$.

Proof. N.C.] Obvious.

S.C.] $E(X)$ is an $A K$-space, therefore $E(X)=S_{(E(X), \tau)}$. Now $S_{\left(\mathcal{A}(X), \tau_{\mathcal{A}}\right)} \subset$ $E(X)$ (proposition 20) and $S_{(E(X), \tau)} \subset S_{\left(\mathcal{A}(X), \tau_{\mathcal{A}}\right)}$, therefore $E(X)=$ $S_{(E(X), \tau)}=S_{\left(\mathcal{A}(X), \tau_{\mathcal{A}}\right)}$, and then $E(X)$ is a closed subspace of $\left(\mathcal{A}(X), \tau_{\mathcal{A}}\right)$. Hence $(E(X), \tau)$ is complete (theorem 11).

\section{References}

[1] R. Ameziane Hassani, M. Babahmed, Topologies polaires compatibles avec une dualité séparante sur un corps valué non-Archimédien, Proyecciones Vol. 20, Núm. 2, pp. 217-240, (2001).

[2] H.R. Chillingworth, Generalised "dual" sequence spaces, Ned. Akad. Proc. Ser. A. 61, pp. 307-515, (1958).

[3] A. El amrani, R. Ameziane Hassani and M. Babahmed, Topologies on sequence spaces in non-archimedean analysis, J. of Mathematical Sciences: Advances and Applications Vol. 6, Núm. 2, pp. 193-214, (2010).

[4] T. Komura; Y. Komura, sur les espaces parfaits de suites et leurs généralisations, J. Math. Soc. Japon. 15, pp. 319-338, (1963).

[5] G. Köthe, Topological vector spaces, Springer-Verlag Berlin Heidlberg New york, (1969).

[6] -----, Neubegründung der theorie der vollkommen Räume, Math. Nach. 4, pp. 70-80, (1951). 
[7] -------; O. Toeplitz, Lineare Raüme mit unendlich vielen Koordinaten und Ringe unendlicher Matrizen, J. reine angew. Math. 171, pp. 193-226, (1934).

[8] G. Matthews, Generalised Rings of infinite matrices, Ned. Akad. Wet. Proc. 61, pp. 298-306 (1958).

[9] A.F.Monna, Analyse non-archimédienne, Springer-Verlag Berlin New York Heidelberg (1970).

[10] H.H. Schaefer, Topological vector spaces, Springer-Verlag Berlin New york Heidlberg, (1971).

[11] W. H. Schikhof, Locally convex spaces over nonspherically complete valued field I, II. Bull. Soc. Math. Belg. Sér. B. 38, pp. 187-224, (1986).

[12] J. Van Tiel, Espaces localement $K$-convexes I-III, Indag. Math. 27, pp. 249-289 (1965).

R. Ameziane Hassani

Département de Mathématiques

Faculté des Sciences Dhar El Mehraz

Université Sidi Mohamed Ben Abdellah

B. P. 1796 FES - MAROC

e-mail : ramezianehassani@hotmail.com

A. El Amrani

Département de Mathématiques

Faculté des Sciences Dhar El Mehraz

Université Sidi Mohamed Ben Abdellah

B. P. 1796, FES - MAROC

e-mail : ramezianehassani@hotmail.com

and

M. Babahmed

Département de Mathématiques

Faculté des Sciences de Meknès

Université Moulay Ismail

B. P. 11201 Zitoune

MEKNES - MAROC

e-mail : babahmed@fs-umi.ac.ma 